\title{
Each portion of fruit or vegetable consumed halves the risk of oral cancer
}

\author{
Does fruit and vegetable intake affect oral cancer?
}

\begin{abstract}
Pavia M, Pileggi C, Nobile CG, Angelillo IF. Association between fruit and vegetable consumption and oral cancer: a meta-analysis of observational studies. Am J Clin Nutr 2006; 83:1126-1134
\end{abstract}

Data sources Medline was used to find relevant papers; reference lists of retrieved publications were also searched.

Study selection Studies were included if they met all of the following criteria: 1) they contained original data from case-control or cohort studies; 2) the primary outcome was clearly defined as cancers of the mouth, pharynx and hypopharynx (as defined by codes 141, 143146,148 and 149 of the ninth revision of the International Classification of Diseases or other classifications that included these sites); 3 ) the exposure of interest was measured as servings (per day, week or month) of fruit, vegetables or both (other exposure measurements, such as mass per day, were excluded unless data were provided to transform information to servings per day); 4) they provided relative risk (RR) estimates and their $95 \%$ confidence intervals $(\mathrm{Cl})$, or sufficient data to calculate these parameters; 5) were published in the English language; and 6) were published before September 2005. If a study appeared in more than one article, data from the most recent publication were used for the statistical analysis. Studies restricted to oral cancer in subjects aged under 45 years were excluded.

Data extraction and synthesis Study quality was assessed and data were extracted independently by two investigators, differences being resolved by discussion. All studies were stratified by fruit or vegetable intake to evaluate any dose-response relations, and some of the studies were used to perform multivariate analyses to adjust for several confounders. Separate meta-analyses were conducted for fruit and vegetable consumption. Potential sources of heterogeneity between the studies were examined using the DerSimonian and Laird method. Sensitivity analyses and a multivariate metaregression analysis were performed to examine observed heterogeneity.

Results A total of 71 potentially relevant studies were identified: 16 were included in the meta-analysis. Seven studies had been carried out in Europe, five in the Americas and four in East Asia. Three case-control studies consisted solely of men and one study consisted solely of women. The pooled data allowed 65802 and 57993 subjects, respectively, to be included in analysis of fruit and vegetable consumption. The combined adjusted odds ratio (OR) estimates based on 16 studies showed that each portion of fruit consumed per day had a statistically significant effect, reducing the risk of oral cancer by $49 \%$ (OR, $0.51 ; 95 \% \mathrm{Cl}$, $0.40-0.65)$. A similar effect was seen for vegetable consumption, with a significant reduction in the risk of oral cancer of 50\% (OR, 0.50; 95\% $\mathrm{Cl}, 0.38-0.65)$. Homogeneity testing found a statistically significant

Address for correspondence: IF Angelillo, Department of Public, Clinical and Preventive Medicine, Second University of Naples, Via Luciano Armanni 5, 80138 Naples, Italy. E-mail: italof.angelillo@unina2.it heterogeneity for fruit consumption and vegetable consumption studies. This was examined using sensitivity analysis which did not substantially modify the main findings of the meta-analysis.

Conclusions The consumption of fruit and vegetables was found to be associated with a reduced risk of oral cancer. Prospective studies are needed to confirm this result.

\section{Commentary}

Oral cancer remains a significant global disease. ${ }^{1}$ Rising incidence across the UK is not entirely explained by trends in the traditional risk factors, namely tobacco smoking and alcohol consumption. ${ }^{2}$ Alternative, or rather, additional factors continue to be investigated, including: diet, human papillomavirus infection, genetics and socioeconomic factors. With a general picture of poor diet in our communities contributing to health issues, the role of food is particularly worthy of exploration in relation to oral cancer.

This study is a significant contribution to reviewing and synthesising the (often conflicting) evidence of the role of diet in developing oral cancer. Building on earlier work begun by the World Cancer Research Fund's ground-breaking systematic review, which found "convincing" evidence that diets high in fruit and vegetables decrease the risk of cancers of the mouth and pharynx,$^{3}$ and continued by an earlier but smaller meta-analysis, ${ }^{4}$ this study goes further. It aims to undertake both a systematic review and meta-analysis of the published literature in the area.

The world of systematic reviewing and meta-analysis generally focuses on analysis of the effectiveness of interventions through pooling evidence from trials (usually randomised control trials) be these clinical (eg, Cochrane collaboration; www.cochrane.org) or social (eg, Campbell collaboration; www.campbellcollaboration.org). Increasingly, however, meta-analysis is being used to synthesise aetiological observational studies (such as case-control or cohort studies) but guidelines do not exist for these studies as they do for systematic reviews on intervention studies. ${ }^{5}$ This is an important paper, therefore, a fact also demonstrated by its use of the following methods: quality assessment of the included case-control and cohort studies; data extraction; meta-analyses, including subgroup analyses; and sensitivity analyses.

Sixteen observational studies (15 case-control and one cohort) from the worldwide literature met the inclusion criteria and provided diet data from nearly 5000 individuals. The main findings from the meta-analysis were that each portion of fruit or vegetables consumed per day reduced the risk of oral cancer by around 50\%.

Interpretation of studies on diet risk factors is extraordinarily difficult because of the various methods and forms of collecting information on diet as well as the general dietary differences between populations. This was reflected in the high level of heterogeneity of the studies included. Nevertheless the thoroughness of the review allows the result to stand. 
The next step in synthesising studies is to pool individual-level data from various studies. The International Head and Neck Cancer Epidemiology Consortium (inhance.iarc.fr/) is indeed beginning to pool data on head and neck cancers from around the world. ${ }^{6}$ This would permit high powered, in-depth and adjusted analysis of risk factors.

Finally, the results of this review do have important public health and nutritional implications, both in terms of developing a better understanding of the aetiology of oral cancer and also for developing prevention approaches. Encouragement of fresh fruit and vegetable consumption should form part of public health and chairside approaches to preventing oral cancer. Changing dietary behaviour is not so easy, however, and the determinants of such behaviour must also be recognised and understood. It is well documented that diet is related to access and affordability of healthy foods, as well as culture and cooking skills. It is not necessarily a simple lifestyle choice. ${ }^{6}$

\section{Practice Point}

The consumption of five portions of fruit and vegetables a day is a key health message that has relevance to oral as well as general health.

\section{David I Conway}

\section{University of Glasgow Dental School, Glasgow, Scotland, UK}

1. Parkin DM, Bray F, Ferlay J, Pisani P. Global cancer statistics, 2002. CA Cancer J Clin 2005; 55:74-108.

2. Conway DI, Stockton DL, Warnakulasuriya KAAS, Ogden G, Macpherson LMD. Incidence of oral and oro-pharyngeal cancer in United Kingdom (1990-1999). Recent trends and regional variation. Oral Oncol 2006; 42:586-592.

3. World Cancer Research Fund and American Institute for Cancer Research. Food Nutrition and the Prevention of Cancer: a Global Perspective. Washington, DC: American Institute for Cancer Research; 1997.

4. Riboli E, Norat T. Epidemiologic evidence of the protective effect of fruit and vegetables on cancer risk. Am J Clin Nutr 2003; 78 (suppl.):S559-S569.

5. Centre for Reviews and Dissemination. Undertaking Systematic Reviews of Research on Effectiveness. CRD Report No. 4 (2nd Edn). York: University of York; 2001.

6. Wrigley N. 'Food Deserts' in British Cities: Policy Context and Research Priorities. Urban Studies 2002; 39:2029-2040.

Evidence-Based Dentistry (2007) 8, 19-20. doi:10.1038/sj.ebd.6400471 